\title{
On effectively computing the analytic center of the solution set by primal-dual interior-point methods*
}

\author{
María D. González-Lima, ${ }^{\dagger}$ Richard A. Tapia, ${ }^{\ddagger}$ and Florian A. Potra ${ }^{\S}$
}

August 14, 1996

\begin{abstract}
The computation of the analytic center of the solution set can be important in linear programming applications where it is desirable to obtain a solution that is not near the relative boundary of the solution set. In this work we discuss the effective computation of the analytic center solution by the use of primal-dual interior-point methods. A primal-dual interior-point algorithm designed for effectively computing the analyticcenter solution is proposed and theory and numerical results are presented.
\end{abstract}

\section{Introduction}

The computation of the analytic-center of the solution set is valuable for some linear programming applications where it is desirable to obtain a solution that is not near the relative

*This research was partially supported by NSF Cooperative Agreement No. CCR-88- 09615, NSF Grant DMS 9305760, ARO Grant 9DAAL03-90-G-0093 DOE Grant DEFG05-86-ER25017, and AFOSR Grant 89-0363.

${ }^{\dagger}$ Departamento de Matemáticas Puras y Aplicadas y Centro de Estadística y Software Matemático (CESMA), Universidad Simón Bolívar, Caracas, Venezuela. Partially supported by Fulbright/LASPAU.

$\ddagger$ Department of Computational and Applied Mathematics and Center for Research on Parallel Computation, Rice University, Houston, TX 77251-1892.

$\S$ Department of Mathematics and Department of Computer Science, The University of Iowa, Iowa City, Iowa 52242 . 
boundary of the solution set (see Charnes, Cooper, and Thrall [3] for such an application). In this work we discuss the use of primal-dual interior-point methods for effectively computing the analytic-center solution.

Because in this work we are interested in using primal-dual interior-point methods for finding a specific solution (the analytic-center solution), the study of the convergence of the iteration sequence generated by primal-dual interior-point methods needs to be addressed. It is relevant to mention that this is not the case for many real-world problems where any feasible point with the value of the duality gap close to zero is a satisfactory approximate solution.

The basic primal-dual interior-point method for linear programming was proposed by Kojima, Mizuno, and Yoshise [11]. Most primal-dual interior-point methods fall into the general framework of the Kojima-Mizuno-Yoshise algorithm. The first work devoted to the study of the convergence of the iteration sequence generated by the Kojima-Mizuno-Yoshise algorithm is Tapia, Zhang, and Ye [18]. Later on, Zhang and Tapia [24] derived conditions under which this iteration sequence converged to the analytic-center solution, assuming that the sequence converged. However, the conditions given in [24] are not compatible with the Tapia-Zhang-Ye conditions for the convergence of the iteration sequencè. The former requires the centering parameters to be bounded away from zero, while the latter requires that these same parameters converge to zero.

An interesting variant of the Kojima-Mizuno-Yoshise algorithm was proposed by Mizuno, Todd, and Ye [16]. The Mizuno-Todd-Ye predictor-corrector algorithm is a path following algorithm which keeps the iterates in a small neighborhood of the central path. Zhang and El-Bakry [22] showed that a modified version of the Mizuno-Todd-Ye predictor-corrector algorithm had the property that the iteration sequence that it generated converged to the analytic-center. Soon after the result of Zhang and El-Bakry, Gonzaga and Tapia [8] proved that the Mizuno-Todd-Ye predictor-corrector algorithm itself, without any modification, generated an iteration sequence which converged to the analytic-center. These are the first results proving convergence of the iteration sequence to the analytic center solution for any primal-dual interior-point method.

The Mizuno-Todd-Ye predictor-corrector algorithm has two characteristics that makes it unappealing for most practical purposes. The first one is that at each iteration the algorithm 
requires the solution of two linear systems of equations where the matrices are of the same form as those for the Kojima-Mizuno-Yoshise algorithm. Hence, the cost per iteration is twice that of the Kojima-Mizuno-Yoshise algorithm. The second deficiency is the requirement that the starting feasible point must be inside a specified $\beta$-neighborhood of the central path.

Recently, Bonnans and Gonzaga [1] gave sufficient conditions for convergence and convergence to the analytic-center of the iteration sequence generated by primal-dual interiorpoint algorithms from a general class of algorithms containing the Kojima-Mizuno-Yoshise and Mizuno-Todd-Ye algorithms. Their result is an extension of the work of Gonzaga and Tapia [8]. We have found that theoretical results are often limited in practice. Theorem 3.2 in Bonnans and Gonzaga [1] guarantees the theoretical convergence of the iteration sequence to the analytic-center assuming, among other conditions, that the iterates stay in a small neighborhood of the central path. However, strong adherence of the iterates to the central path results in slow performance of the algorithm, especially when this adherence is also maintained far from the solution set. In addition, we will show that although the iteration sequence generated by the Kojima-Mizuno-Yoshise algorithm may theoretically converge to the analytic-center, in practice this convergence is not, for many cases, easy to attain. This negative behavior is related to the proximity of the iterates to the solution set. If the sequence generated by the algorithm approaches the solution set very fast and is far from the analytic-center the stopping criteria are met before the approximate solution has a chance of getting close to the analytic-center. Also, the singularity of the Jacobian matrix at the solution may cause serious numerical problems that affect the computation of the analytic center when the iterates are very close to the solution set.

Hence, a balance between proximity to the central path and proximity to the solution set is crucial for an effective computation of the analytic-center of the solution set using a primal-dual interior-point algorithm.

In this work a modification of the Kojima-Mizuno-Yoshise primal-dual algorithm is proposed. This modified algorithm combines the objectives of approaching the central path and the solution set to effectively compute the analytic-center solution. We prove that the proposed algorithm generates an iteration sequence with the property that a subsequence converges to the analytic-center of the solution set; however in practice we always have that the entire sequence converges to the analytic center. Moreover, we also prove that with a 
small modification the algorithm achieves polynomial complexity. Finally, we demonstrate that the numerical behavior of the algorithm is quite good.

This paper is organized as follows. The next section contains some preliminary background and notation. In Section 3 our proposed primal-dual interior-point algorithm designed for effectively computing the analytic-center is described. The convergence properties for the algorithm are studied in Section 4. In Section 5 we prove that the algorithm has polynomial complexity if the algorithm is modified in an appropriate way. In Section 6 we study the numerical behavior of the proposed algorithm. Also, we compare the numerical behavior of the proposed algorithm with the numerical behavior of the Kojima-Mizuno-Yoshise and the Mizuno-Todd-Ye algorithms. Finally, some conclusions and remarks are given in Section 7.

\section{Preliminaries and Notation}

We consider the linear programming problem in the standard form

$$
\begin{array}{ll}
\text { minimize } & c^{T} x \\
\text { subject to } & A x=b, \quad x \geq 0,
\end{array}
$$

where $c, x \in \mathbf{R}^{n}, b \in \mathbf{R}^{m}, A \in \mathbf{R}^{m \times n}(m<n)$.

The Karush-Kuhn-Tucker optimality conditions for (1) are

$$
F(x, y, z)=\left(\begin{array}{c}
A x-b \\
A^{T} y+z-c \\
X Z e
\end{array}\right)=0, \quad(x, z) \geq 0
$$

where $X=\operatorname{diag}(x), Z=\operatorname{diag}(z)$, and $e=(1,1, \ldots 1,1)^{T} \in \mathbf{R}^{n}$.

The feasibility set of problem (2) is

$$
\mathcal{F}=\left\{(x, y, z): A x=b, A^{T} y+z=c,(x, z) \geq 0\right\}
$$

A feasible point $(x, y, z) \in \mathcal{F}$ is said to be strictly feasible if $x$ and $z$ are strictly positive. The set of all the strictly feasible points is denoted by $\mathcal{F}^{+}$. 
We denote the solution set of problem (2) by

$$
\mathcal{S}=\{(x, y, z): F(x, y, z)=0,(x, z) \geq 0\}
$$

A point $(x, y, z)$ in $\mathcal{S}$ is said to be a strict complementarity solution if $x_{i}+z_{i}>0$ for all $i=1, \ldots, n$.

The following standard conditions are assumed:

(A1) $\left\{x \in \mathbf{R}^{n}: A x=b, x>0\right\} \neq \emptyset$, and

(A2) $\left\{(y, z) \in \mathbf{R}^{m+n}: A^{T} y+z=c, z>0\right\} \neq \emptyset$.

(A3) $A$ has full rank $m$.

We are particularly concerned with the case when $\mathcal{S}$ is not a singleton set.

Under the previous assumptions, the solution set $\mathcal{S}$ has the following interesting structure:

(i) The set $\mathcal{S} \neq \emptyset$ is bounded.

(ii) All points in the relative interior of $\mathcal{S}$ are strict complementarity solutions and all points on the relative boundary of $\mathcal{S}$ are not.

(iii) The zero-nonzero pattern of points in the relative interior of $\mathcal{S}$ is invariant.

See El-Bakry, Tapia, and Zhang [6] for proofs.

Therefore, for any $\left(x^{*}, y^{*}, z^{*}\right) \in \operatorname{ri}(\mathcal{S})$, where $\operatorname{ri}(\mathcal{S})$ denotes the relative interior of $\mathcal{S}$, the following index sets

$$
I_{x}^{+}=\left\{i: x_{i}^{*}>0,1 \leq i \leq n\right\} \text { and } I_{z}^{+}=\left\{i: z_{i}^{*}>0,1 \leq i \leq n\right\}
$$

are independent of the choice of $\left(x^{*}, y^{*}, z^{*}\right)$. Moreover, by strict complementarity

$$
I_{x}^{+} \bigcup I_{z}^{+}=\{1,2, \ldots, n\} \text { and } I_{x}^{+} \bigcap I_{z}^{+}=\emptyset
$$

Because of this structure of the solution set $\mathcal{S}$, the solutions in $\operatorname{ri}(\mathcal{S})$ may be characterized as having a maximal number of nonzero components. Among them, there is a solution that may be thought of as the center-most solution in the sense that it maximizes the product of 
the positive components. This solution is called the analytic-center of the solution set and was studied by McLinden [13] in a general setting and later independently by Sonnevend [17] in the context of linear programming. Formally, the analytic-center of the solution set $\mathcal{S}$ is defined as:

$$
\left(x^{*}, y^{*}, z^{*}\right)=\arg \max \{\psi(x, z):(x, y, z) \in \mathcal{S}\}
$$

where

$$
\psi(x, z)=\prod_{i \in I_{x}^{+}} x_{i} \prod_{i \in I_{z}^{+}} z_{i} .
$$

Equivalently, in (3) one can replace $\psi(x, z)$ by its logarithm, i.e.,

$$
\ln \psi(x, z)=\sum_{i \in I_{x}^{+}} \ln x_{i}+\sum_{i \in I_{z}^{+}} \ln z_{i} .
$$

The central path associated with problem (2) parametrized by the parameter $\mu$ is defined as the collection of points $\mathcal{P}_{c}=\left\{\mathcal{P}_{c}(\mu) ; \mu>0\right\}$ where

$$
\mathcal{P}_{c}(\mu)=(x(\mu), y(\mu), z(\mu)) \text { is a strictly feasible point satisfying } X(\mu) Z(\mu) e=\mu e .
$$

This is equivalent to saying that a strictly feasible point $(x, y, z)$ is on the central path (for some $\mu>0)$ if and only if it satisfies $x_{1} z_{1}=x_{2} z_{2}=\ldots=x_{n} z_{n}$.

These notions of analytic-center and central path are well-defined under assumptions (A1), and (A2). For more details see McLinden [13], Megiddo [15], and Gonzaga [7].

A $\beta$-neighborhood of the central path is defined as

$$
\mathcal{N}(\beta)=\left\{(x, y, z):(x, y, z) \in \mathcal{F},\|X z-\mu e\|_{2} \leq \beta \mu\right\}
$$

where $\beta \in(0,1)$ and $\mu=x^{T} z / n$.

A very interesting result is Theorem 9 of McLinden [13]. In the case of linear programming, it states that the central path intersects the solution set at the analytic-center, i.e., the central-path point $(x(\mu), y(\mu), z(\mu))$ converges to the analytic center $\left(x^{*}, y^{*}, z^{*}\right)$ as $\mu$ converges to zero. See also Proposition 8.2 in Megiddo [15] and the discussion preceding it. This fact plays a critical role in the development of most primal-dual interior-point algorithms which attempt to follow the central path. 


\section{Algorithm}

The interior-point algorithm proposed in this work has been designed for effectively computing the analytic-center of the solution set in linear programming and it is a modification of the Kojima-Mizuno-Yoshise algorithm.

The following lemma can be found in Zhang and Tapia [24]. It provides a sufficient condition for a strictly feasible sequence $\left\{\left(x^{k}, y^{k}, z^{k}\right)\right\}$ to converge to the analytic-center of the solution set.

Lemma 3.1 (Zhang-Tapia) Let $\left\{\left(x^{k}, y^{k}, z^{k}\right)\right\}$ be a sequence of strictly feasible points. Let (a1)

$$
\mu^{k}=\left(x^{k}\right)^{T} z^{k} / n \rightarrow 0
$$

and

(a2)

$$
\left\|X^{k} z^{k} / \mu^{k}-e\right\|_{2} \rightarrow 0
$$

with $e=(1, \ldots, 1)^{T} \in \mathbf{R}^{n}$.

Then $\left\{\left(x^{k}, y^{k}, z^{k}\right)\right\}$ converges to the analytic-center of the solution set.

Our approach to constructing an algorithm for computing the analytic center is to attempt to enforce conditions (5) and (6).

A key ingredient in the proposed algorithm is the use of the damped Newton method applied to the perturbed Karush-Kuhu-Tucker conditions

$$
F_{\mu}(x, y, z)=\left(\begin{array}{c}
A x-b \\
A^{T} y+z-c \\
X Z e-\mu e
\end{array}\right)=0
$$

with $\mu>0$. A line-search technique is used as a globalization of Newton's method. For fixed $\boldsymbol{\mu}>\mathbf{0}$, the merit function considered is $f_{\mu}: \mathbf{R}^{2 n+m} \rightarrow \mathbf{R}$ defined by

$$
f_{\mu}(x, y, z)=\left\|F_{\mu}(x, y, z) / \mu\right\|_{2}^{2}
$$


Observe that

$$
f_{\mu}(x, y, z)=\|(X z-\mu e) / \mu\|_{2}^{2}, \text { for }(x, y, z) \in \mathcal{F}
$$

The function $f_{\mu}$ measures proximity or closeness to the central path at $\mu$.

The basic idea of the algorithm is to consider a sequence of gradually shrinking neighborhoods $\left\{\mathcal{N}\left(\beta^{j}\right)\right\}$ of the central path with $\beta^{j} \rightarrow 0$. In the next section we will show that under certain assumptions the proposed algorithm generates an iteration sequence $\left\{\left(x^{k}, y^{k}, z^{k}\right)\right\}$ such that a subsequence $\left(x^{k_{j}}, y^{k_{j}}, z^{k_{j}}\right)$ belongs to $\mathcal{N}\left(\beta^{j}\right)$. This subsequence is obtained by considering a sequence of $\mu^{\prime} s$ and for each fixed $\mu$ using a damped Newton's method (with a line-search globalization strategy) to approximately solve the system $\mathcal{F}_{\mu}(x, y, z)=0$.

A description of the algorithm is the following:

\section{Algorithm 3.1 (Long-Step Shrinking-Neighborhood (LSSN) Algorithm) Consider} a strictly feasible point $w^{0}=\left(x^{0}, y^{0}, z^{0}\right), \sigma^{0} \in(0,1)$, and $\beta^{0} \in(0,1)$.

Choose $\eta \in(0,1 / 2)$ and $0<l<u<1$.

Do until convergence

(0) Set $k=0$.

(1) Define parameters: Set $\mu=\mu^{k}=\sigma^{0}\left(x^{k}\right)^{T} z^{k} / n$ and $\beta=\beta^{k}$.

(2) Check proximity to the central path: If

$$
\left\|X^{k} Z^{k} e / \mu-\epsilon\right\|_{2} \leq \beta
$$

go to (6).

(3) Compute new iterate:

(3.1) Solve the following system for $\Delta w^{k}=\left(\Delta x^{k}, \Delta y^{k}, \Delta z^{k}\right)$ :

$$
F^{\prime}\left(x^{k}, y^{k}, z^{k}\right)\left(\begin{array}{c}
\Delta x \\
\Delta y \\
\Delta z
\end{array}\right)=-F\left(x^{k}, y^{k}, z^{k}\right)+\mu\left(\begin{array}{l}
0 \\
0 \\
e
\end{array}\right) \text {. }
$$


(3.2) Choose $\tau^{k} \in(0,1)$ and compute the step-length $\alpha^{k}=\min \left(1, \tau^{k} \hat{\alpha}^{k}\right)$, where

$$
\hat{\alpha}^{k}=\frac{-1}{\min \left(\left(X^{k}\right)^{-1} \Delta x^{k},\left(Z^{k}\right)^{-1} \Delta z^{k}\right)} .
$$

(3.3) Form the iterate

$$
w^{k+1}=\left(x^{k+1}, y^{k+1}, z^{k+1}\right)=\left(x^{k}, y^{k}, z^{k}\right)+\alpha^{k}\left(\Delta x_{2}^{k}, \Delta y^{k}, \Delta z^{k}\right) .
$$

(4) Line search:

(4.1) If

$$
f_{\mu}\left(w^{k+1}\right) \leq f_{\mu}\left(w^{k}\right)+\eta \alpha^{k} \nabla f_{\mu}\left(w^{k}\right)^{T}\left(\Delta x^{k}, \Delta y^{k}, \Delta z^{k}\right)^{T}
$$

go to (5).

(4.2) If not, reduce $\alpha^{k}:=\rho \alpha^{k}$, with $\rho \in[l, u]$ and form the iterate

$$
w^{k+1}=\left(x^{k+1}, y^{k+1}, z^{k+1}\right)=\left(x^{k}, y^{k}, z^{k}\right)+\alpha^{k}\left(\Delta x^{k}, \Delta y^{k}, \Delta z^{k}\right) .
$$

(4.3) Go to step (4.1).

(5) Set $k:=k+1, \beta^{k}=\beta, \mu^{k}=\mu$ and go to (2).

(6) Set $\mu=\mu^{k}=\sigma^{0}\left(x^{k}\right)^{T} z^{k} / n$.

(7) $D_{0}(3.1),(3.2),(3.3)$.

(8) Decrease $\beta$-neighborhood: Choose $\beta^{k+1} \leq \beta^{k}$.

(9) Set $k:=k+1$ and go to (1).

We note that while condition (8) is not satisfied substeps (3) and (4) of the above algorithm are performed iteratively with the same value of $\mu$. It is convenient to denote by $\mathcal{I}(\mu)$ the corresponding set of indices i.e.,

$$
\mathcal{I}(\mu)=\left\{k: \mu^{k}=\mu\right\}
$$

In the next section we will show that the index set $\mathcal{I}(\mu)$ is finite for each $\mu$ chosen by Algorithm 3.1. This means that under certain assumptions the iteration sequence generated by the LSSN algorithm, when $\mu>0$ is fixed, approaches the central path in the sense that condition (8) (or proximity to the central path) is satisfied. 


\section{Convergence Properties}

In this section we prove that under certain assumptions the LSSN algorithm generates an iteration sequence which contains a subsequence that converges to the analytic-center of the solution set. The convergence result is established by showing that under certain assumptions there exists a subsequence satisfying conditions (5) and (6) of Lemma 3.1. Therefore we need to study the behavior of the proposed algorithm with respect to the two objectives of decreasing the gap and centering the iterates. In the following two propositions we study these issues. In the first proposition we prove that the gap sequence generated by the LSSN algorithm is a decreasing sequence. In the second proposition we prove that for each fixed $\mu>0$, the search direction is a descent direction for the function $f_{\mu}$ and the sequence $\left\{f_{\mu}\left(x^{k}, y^{k}, z^{k}\right)\right\}$ generated by the algorithm is a strictly decreasing sequence. Also, we show that $f_{\mu}\left(x^{k}, y^{k}, z^{k}\right)$ is an upper bound for the Euclidean measure $\left\|\frac{X^{k} z^{k}}{\left(x^{k}\right)^{T} z^{k} / n}-e\right\|_{2}^{2}$, therefore condition (6) may be established for any sequence of iterates $\left\{\left(x^{k}, y^{k}, z^{k}\right)\right\}$ with the property that the corresponding sequence $\left\{f_{\mu}\left(x^{k}, y^{k}, z^{k}\right)\right\}$ converges to zero.

Proposition 4.1 Let $\left\{\left(x^{k}, y^{k}, z^{k}\right)\right\}$ and $\left\{\alpha^{k}\right\}$ be generated by the LSSN Algorithm (Algorithm 3.1). Then,

$$
\left(x^{k+1}\right)^{T} z^{k+1}=\left(1-\alpha^{k}\left(1-\sigma^{k}\right)\right)\left(x^{k}\right)^{T} z^{k}, \text { for all } k \geq 0 \text { and for some } \sigma^{k} \in(0,1] \text {. }
$$

\section{Proof}

Define the following sets

$$
\begin{gathered}
I_{c p}=\left\{k \geq 0:\left(x^{k}, y^{k}, z^{k}\right) \text { satisfies condition (8) in the algorithm }\right\}, \text { i.e. } \\
I_{c p}=\left\{k \geq 0:\left\|X^{k} Z^{k} e / \mu-e\right\|_{2} \leq \beta^{k}\right\}, \text { and } . \\
I_{c p}^{+}=\left\{k \geq 1:\left(x^{k-1}, y^{k-1}, z^{k-1}\right) \text { satisfies condition (8) in the algorithm }\right\} \cup\{0\} .
\end{gathered}
$$

For each $k$ we denote by $k_{c p}^{+}$the largest element in $I_{c p}^{+}$such that $k_{c p}^{+} \leq k$ and $k_{c p}$ the smallest element in $I_{c p}$ such that $k \leq k_{c p}$.

Let $\sigma^{k}$ for all $k \geq 0$ be defined as

$$
\sigma^{k}=\sigma^{o} / \prod_{m=k_{c p}^{+}}^{k-1}\left(1-\alpha^{m}\left(1-\sigma^{m}\right)\right), \text { for } k \notin I_{c p} \cup I_{c p}^{+}
$$


and

$$
\sigma^{k}=\sigma^{o}, \text { for } k \in I_{c p} \cup I_{c p}^{+} .
$$

A fairly lengthy but straightforward inductive proof can be used to establish the following results (a detailed proof can be found in González-Lima [9])

(i) $\sigma^{k} \in(0,1]$, for all $k \geq 0$.

(ii) $\sigma^{k}=1$ if $\alpha^{k-1}=1$, for $k \notin I_{c p} \cup I_{c p}^{+}$.

(iii) $\sigma^{k_{c p}^{+}+1} \leq \sigma^{k_{c p}^{+}+2} \leq \ldots \leq \sigma^{k_{c p}} \leq 1$.

From step (3.1) in the algorithm we have

$$
\left(x^{k+1}\right)^{T} z^{k+1}=\left(x^{k}\right)^{T} z^{k}\left(1-\alpha^{k}\right)+n \alpha^{k} \mu, \text { for all } k \geq 0 .
$$

Then, the remainder of the proof consists in showing that

$$
\mu=\sigma^{k}\left(x^{k}\right)^{T} z^{k} / n, \text { for all } k \geq 0 .
$$

If $k \in I_{c p} \cup I_{c p}^{+}$then $\sigma^{k}=\sigma^{0}$, and by the construction of the algorithm we have

$$
\mu=\sigma^{0} \frac{\left(x^{k}\right)^{T} z^{k}}{n}=\sigma^{k} \frac{\left(x^{k}\right)^{T} z^{k}}{n} .
$$

If $k \notin I_{c p} \cup I_{c p}^{+}$, assume without loss of generality that $k_{c p}^{+}=0$ and $k=2$.

From (11) we get

$$
\left(x^{2}\right)^{T} z^{2}=\left(x^{1}\right)^{T} z^{1}\left(1-\alpha^{1}\right)+n \alpha^{1} \mu .
$$

Multiplying both sides of equation (13) by $\sigma^{2} / n$, using the definition of $\sigma^{2}$ and the fact that

$$
\mu=\sigma^{1} \frac{\left(x^{1}\right)^{T} z^{1}}{n},
$$

we obtain

$$
\mu=\sigma^{2} \frac{\left(x^{2}\right)^{T} z^{2}}{n} .
$$

The use of the same argument in an inductive manner for $k \leq k_{c p}$ completes the proof of (12). 
Proposition 4.2 Let $\mu>0$ be a parameter selected by Algorithm 3.1 and consider the index set $\mathcal{I}(\mu)$ defined by (10). Then the following properties are satisfied for any $k \in \mathcal{I}(\mu)$ :

(i) $\Delta w^{k}$ is a descent direction for the merit function

$$
f_{\mu}(x, y, z)=\left\|\frac{X Z e-\mu e}{\mu}\right\|_{2}^{2}
$$

$$
\text { at } w^{k}=\left(x^{k}, y^{k}, z^{k}\right) .
$$

(ii) $f_{\mu}\left(w^{k+1}\right) \leq\left(1-\eta \alpha^{k}\right) f_{\mu}\left(w^{k}\right)$, where $\eta \in(0,1)$ is chosen as in the algorithm.

(iii) $\left\|\frac{X^{k} Z^{k}}{\left(x^{k}\right)^{T} z^{k} / n} e-e\right\|_{2} \leq f_{\mu}\left(w^{k}\right)^{1 / 2}$.

Proof.

Since $w^{k} \in \mathcal{F}^{+}$we have

$$
\begin{aligned}
f_{\mu}\left(w^{k}\right) & =\frac{1}{\mu^{2}}\left\|F_{\mu}\left(w^{k}\right)\right\|_{2}^{2} \text { and } \\
\nabla f_{\mu}\left(w^{k}\right) & =\frac{2}{\mu^{2}}\left(F^{\prime}\left(w^{k}\right)\right)^{T} F_{\mu}\left(w^{k}\right) .
\end{aligned}
$$

Therefore,

$$
\nabla f_{\mu}\left(w^{k}\right)^{T} \Delta w^{k}=-\frac{2}{\mu^{2}} F_{\mu}^{T}\left(w^{k}\right) F_{\mu}\left(w^{k}\right)=-\frac{2}{\mu^{2}}\left\|F_{\mu}\left(w^{k}\right)\right\|_{2}^{2}<0
$$

and (i) follows.

Part (ii) can be demonstrated in a straightforward manner from condition (9) of the algorithm.

Now, let us prove part (iii). Because $\frac{\left(x^{k}\right)^{T} z^{k}}{n} e$ is the orthogonal projection of $X^{k} Z^{k} e$ over the subspace generated by $e$ we have

$$
\left\|X^{k} Z^{k} e-\frac{\left(x^{k}\right)^{T} z^{k}}{n} e\right\|_{2} \leq\left\|X^{k} Z^{k} e-\mu e\right\|_{2}=\mu f_{\mu}\left(w^{k}\right)^{1 / 2},
$$

for all $k$.

Hence,

$$
\left\|\frac{X^{k} Z^{k} e}{\left(x^{k}\right)^{T} z^{k} / n}-e\right\|_{2} \leq f_{\mu}\left(w^{k}\right)^{1 / 2}\left(\frac{\mu}{\left(x^{k}\right)^{T} z^{k} / n}\right) .
$$


By Proposition 4.1, $\mu=\sigma^{k}\left(x^{k}\right)^{T} z^{k} / n$, with $\sigma^{k} \in(0,1]$. Therefore

$$
\left\|\frac{X^{k} Z^{k} e}{\left(x^{k}\right)^{T} z^{k} / n}-e\right\|_{2} \leq f_{\mu}\left(w^{k}\right)^{1 / 2} .
$$

Moreover, if $\sigma^{k}=1$ the previous inequality is satisfied as an equality.

The next lemma is needed for proving that under mild assumptions, condition (8) from the algorithm (proximity to the central path) is always satisfied.

Lemma 4.2 Let $\mu>0$ be a parameter selected by Algorithm 3.1. and assume that

$$
\min \left(X^{k} Z^{k} e\right) / x^{k^{T}} z^{k} \geq \gamma / n \text { for all } k \in \mathcal{I}(\mu) \text { and some } \gamma \in(0,1),
$$

where $\mathcal{I}(\mu)$ is the index set defined by (10). Then,

$$
\left\|\left(\Delta x^{k}, \Delta y^{k}, \Delta z^{k}\right)\right\|_{2} \leq M<+\infty
$$

for all $k \in \mathcal{I}(\mu)$ and some $M>0$.

\section{Proof.}

Suppose $\left(x^{k}\right)^{T} z^{k} \geq \epsilon$ for some $\epsilon>0$ and for all $k \geq 0$. By hypothesis, $x_{i}^{k} z_{i}^{k} \geq \gamma \frac{\left(x^{k}\right)^{T} z^{k}}{n}$, hence

$$
x_{i}^{k} z_{i}^{k} \geq \frac{\gamma \epsilon}{n} \text { for all } k \geq 0 \text { and } i=1, \cdots, n .
$$

Let $\mathcal{F}_{o}=\left\{(x, y, z) \in \mathcal{F}: x^{T} z \leq\left(x^{0}\right)^{T} z^{0}\right\}$. This set is bounded (see the proof of Lemma 2.1 in Zhang and Tapia [24]). Moreover, by Proposition 4.1, the iterates $\left(x^{k}, y^{k}, z^{k}\right) \in \mathcal{F}_{o}$. Therefore, equation (14) implies that $\left\{x^{k}\right\}$ and $\left\{z^{k}\right\}$ are bounded away from zero for all $k \geq 0$.

Let us denote $w^{k}=\left(x^{k}, y^{k}, z^{k}\right)$ and $\Delta w^{k}=\left(\Delta x^{k}, \Delta y^{k}, \Delta z^{k}\right)$. It follows from the boundedness of the sequences $\left\{x^{k}\right\}$ and $\left\{z^{k}\right\}$ that there exist constants $C$ and $M_{1}$ such that

$$
\left\|\left(F^{\prime}\left(w^{k}\right)\right)^{-1}\right\|_{2}=\left\|\left(\left[\begin{array}{ccc}
A & 0 & 0 \\
0 & A^{T} & I \\
Z^{k} & 0 & X^{k}
\end{array}\right]\right)^{-1}\right\|_{2} \leq C
$$

and

$$
\left\|\Delta u^{k}\right\|_{2} \leq\left\|\left(F^{\prime}\left(w^{k}\right)\right)^{-1}\right\|_{2}\left\|F_{\mu}\left(w^{k}\right)\right\|_{2} \leq M_{1} .
$$


Now, suppose that $\left(x^{k}\right)^{T} z^{k} \rightarrow 0$. By Theorem 3.2 from Tapia, Zhang, and Ye [18] we have that

$$
\left\|\left(\Delta x^{k}, \Delta z^{k}\right)\right\|_{2} \leq C_{1} \sigma^{k}+C_{2}\left(x^{k}\right)^{T} z^{k}
$$

for some constants $C_{1}, C_{2}>0$, and $\sigma^{k}$ defined as in Proposition $4.1\left(\sigma^{k} \leq 1\right)$.

Equation (16) implies that there exists $N_{1}>0$ such that

$$
\left\|\left(\Delta x^{k}, \Delta z^{k}\right)\right\|_{2} \leq N_{1} \text { for all } k \geq 0
$$

Since we are assuming that $\operatorname{rank}(\mathrm{A})=\mathrm{m}$, the matrix $A A^{T}$ is invertible and

$$
\Delta y^{k}=-\left(A A^{T}\right)^{-1} A \Delta z^{k}
$$

Therefore, (17) and (18) imply that there exists $N_{2}>0$ such that

$$
\left\|\Delta y^{k}\right\|_{2} \leq N_{2} \text { for all } k \geq 0
$$

The final result follows from (15), (17), and (19).

The next lemma states that under some natural assumptions the index sets $\mathcal{I}(\mu)$ are finite for any $\mu>0$ generated by the LSSN algorithm.

Lemma 4.3 Suppose in the LSSN Algorithm (Algorithm 3.1) the parameter choice $\left\{\tau^{k}\right\}$ has been made so that the following condition is satisfied:

(c1) $\tau^{k} \geq \tau$ for all $k$ and some $\tau \in(0,1)$.

Now, assume that the points generated by the LSSN Algorithm satisfy

(a1) $\min \left(X^{k} Z^{k} e\right) / x^{k^{T}} z^{k} \geq \gamma / n$ for all $k$ and some $\gamma \in(0,1)$.

Then, the index sets $\mathcal{I}(\mu)$ defined by (10) are finite for any value of $\mu$ selected by the algorithm.

Proof. Assume by contradiction that Algorithm 3.1 selects a value of $\mu$ such that $\mathcal{I}(\mu)$ is infinite i.e.,

$$
\mathcal{I}(\mu)=\{N, N+1, N+2, \ldots,\}
$$


for some $N>0$. Under this assumption we will prove that

$$
f_{\mu}\left(w^{k}\right) \rightarrow 0
$$

where $f_{\mu}$ is defined by (7) and $w^{k}=\left(x^{k}, y^{k}, z^{k}\right)$. It is easily seen that (20) implies that condition (8) will be satisfied for $k$ large enough which contradicts the assumption that $\mathcal{I}(\mu)$ is infinite.

Let us call $\bar{\alpha}^{k}=\min \left(1, \tau^{k} \hat{\alpha}^{k}\right)$, where

$$
\hat{\alpha}^{k}=\frac{-1}{\min \left(\left(X^{k}\right)^{-1} \Delta x^{k},\left(Z^{k}\right)^{-1} \Delta z^{k}\right)}, \text { and } \tau^{k} \in(0,1) \text {. }
$$

It is known (see the proof of Theorem 3.1 in Zhang, Tapia, and Dennis [25]) that Condition (c1) and Assumption (a1) imply that $\bar{\alpha}^{k}$ is bounded away from zero.

If $\alpha^{k}=\bar{\alpha}^{k}$ in infinitely many iterations, then by using (ii) of Proposition 4.2 we deduce immediately that (20) holds. Now, suppose that $\alpha^{k}<\bar{\alpha}^{k}$ for $k$ sufficiently large. It is known (e.g. see Theorem 6.3.3 in Dennis and Schuabel [4] and the consequent discussion) that the backtracking line search used in the LSSN algoritlım (Algoritlım 3.1) guarantees that

$$
\frac{\nabla f_{\mu}\left(w^{k}\right)^{T} \Delta w^{k}}{\left\|\Delta w^{k}\right\|_{2}} \rightarrow 0
$$

where $\Delta w^{k}=\left(\Delta x^{k}, \Delta y^{k}, \Delta z^{k}\right)$. According to the proof of Proposition 4:2 we can write

$$
\nabla f_{\mu}\left(w^{k}\right)^{T} \Delta w^{k}=\frac{-2}{\mu^{2}}\left\|F_{\mu}\left(w^{k}\right)\right\|_{2}^{2}=-2 f_{\mu}\left(w^{k}\right)
$$

and by virtue of Lemma 4.2 , we have that $\left\|\Delta w^{k}\right\|_{2} \leq M$ for all $k$. Therefore, equations (21) and (22) imply that (20) is satisfied in this case as well. This completes the proof of our lemma.

Using Lemma 4.3 we will show that if the parameters $\beta^{j}$ that define the neighborhoods of the central path are chosen so that they converge to zero, then the iteration sequence generated by Algorithm 3.1 possesses a subsequence converging to the analytic-center of the solution set. The next theorem formally states this result.

Theorem 4.1 Let $\left\{\left(x^{k}, y^{k}, z^{k}\right)\right\}$ be generated by the LSSN algorithm (Algorithm 3.1) with parameter choices $\sigma^{0} \in(0,1),\left\{\beta^{k}\right\}$, and $\left\{\tau^{k}\right\}$. Suppose the parameter choices have been made so that the following two conditions are satisfied: 
(c1) $\tau^{k} \geq \tau>0$ for all $k$ and some $\tau \in(0,1)$.

(c2) $\beta^{k} \rightarrow 0$.

Assume

(a1) $\min \left(X^{k} Z^{k} e\right) / x^{k^{T}} z^{k} \geq \gamma / n$ for all $k$ and some $\gamma \in(0,1)$.

Then, there exists a subsequence of $\left\{\left(x^{k}, y^{k}, z^{k}\right)\right\}$ that converges to the analytic-center of the solution set.

Proof.

By Assumption (a1), Condition (c1), and Lemma 4.3, there exists a subsequence $\left\{k^{j}\right\}$ such that (8) is satisfied at iteration $k^{j}$ i.e.,

$$
\left\|\frac{X^{k_{j}} Z^{k_{j}} e}{\mu^{k_{j}}}-e\right\|_{2} \leq \beta^{k_{j}} .
$$

According to (iii) of Proposition (4.2) we have,

$$
\left\|\frac{X^{k_{j}} Z^{k_{j}} e}{\left(x^{k_{j}}\right)^{T} z^{k_{j}} / n}-\epsilon\right\|_{2} \leq\left(f_{\mu}\left(w^{k_{j}}\right)\right)^{1 / 2}
$$

and by virtue of Condition (c2) it follows that

$$
\left\|\frac{X^{k_{j}} Z^{k_{j}} e}{\left(x^{k_{j}}\right)^{T} z^{k_{j}} / n}-e\right\|_{2} \rightarrow 0
$$

Now, for each $j>0$, Proposition (4.1) implies that $\left(x^{k_{j+1}}\right)^{T} z^{k_{j+1}} \leq\left(x^{k_{j+1}}\right)^{T} z^{k_{j+1}}$ and $\left(x^{k_{j}+1}\right)^{T} z^{k_{j+1}} \leq \zeta\left(x^{k_{j}}\right)^{T} z^{k_{j}}$ for some $\zeta \in(0,1)$. Therefore,

$$
\left(x^{k_{j}}\right)^{T} z^{k_{j}} \rightarrow 0 .
$$

The remainder of the proof follows from (23), (24), and Lemma 3.1.

\section{Complexity Bound}

In this section we address two important issues concerning the LSSN algorithm; namely the centrality assumption in Theorem 4.1 and the complexity of the algorithm. We prove that 
a modification of the line-search strategy for the LSSN algorithm leads to a modified LSSN algorithm which possesses polynomial complexity and for which the centrality assumption of Theorem 4.1 is always satisfied.

In order to facilitate the exposition below let

$$
\mathcal{F}_{\gamma}=\left\{(x, y, z) \text { strictly feasible }: \frac{\min (X Z e)}{x^{T} z / n} \geq \gamma\right\}, \text { for } \gamma \in(0,1) .
$$

Clearly if $\left(x^{k}, y^{k}, z^{k}\right) \in \mathcal{F}_{\gamma}$, for all $k \geq 0$, then the sequence $\left\{\left(x^{k}, y^{k}, z^{k}\right)\right\}$ satisfies Assumption (a1) (the centrality assumption) of Theorem 4.1 .

For each $\mu>0$, the modified LSSN algorithm presented in this section considers an exact line-search instead of the backtracking strategy performed by the LSSN algorithm. The exact line-search is defined so that the iterates generated by the modified LSSN algorithm always belong to the set $\mathcal{F}_{\gamma}$. Therefore, we demonstrate that the assumption of Theorem 4.1 can always be enforced. Moreover, since the merit function for the line-search is the Euclidean norm proximity measure given by $f_{\mu}$, a polynomial bound can be derived for the number of iterations required by the algorithm to satisfy the proximity condition to the central path given by (8). Hence, polynomial complexity is attainable.

The following is a description of the modified LSSN algorithm.

\section{Algorithm 5.2 (Modified Long-Step Shrinking-Neighborhood (LSSN) Algorithm)} Consider a strictly feasible point $w^{0}=\left(x^{0}, y^{0}, z^{0}\right), \sigma^{0} \in(0,1)$ and $\beta^{0} \in(0,1)$. Choose $\gamma \in(0,2-\sqrt{2}]$ such that $\gamma \leq \frac{\min \left(X^{0} Z^{0} e\right)}{\left(x^{0}\right)^{T} z^{0} / n}$.

Do until convergence

(0) Set $k=0$.

(1) Set $\mu^{k}=\sigma^{0}\left(x^{k}\right)^{T} z^{k} / n$.

(2) Set $\mu=\mu^{k}$ and $\beta=\beta^{k}$.

(3) Denote $w^{k, 0}=w^{k}$.

(4) Set l $=0$.

(5) Set $w=(x, y, z)=w^{k, l}$. 
(6) Check proximity to the central path: If

$$
\|X Z e / \mu-e\|_{2} \leq \beta
$$

go to $(\mathbf{1 0})$.

(7) Compute new iterate:

(7.1) Solve the following system for $\Delta u=(\Delta x, \Delta y, \Delta z)$ :

$$
F^{\prime}(x, y, z)\left(\begin{array}{c}
\Delta x \\
\Delta y \\
\Delta z
\end{array}\right)=-F(x, y, z)+\mu\left(\begin{array}{l}
0 \\
0 \\
e
\end{array}\right) .
$$

(7.2) Line search: Find the solution $\bar{\theta}$ of

$$
\min _{w+\theta \Delta w \in \mathcal{F}_{\gamma}} f(\theta),
$$

where $f(\theta)=\|X(\theta) z(\theta) / \mu-\epsilon\|_{2}$.

(7.3) Form the iterate $w^{+}=w+\bar{\theta} \Delta u$.

(8) Set $w^{k, l+1}=w^{+}$and $\theta^{k, l}=\bar{\theta}$.

(9) Set $l=l+1$ and go to (5).

(10) Set $l^{k}=l$ and $w^{k+1}=w^{k, l^{k}}$.

(11) Decrease $\beta$-neighborhood: Choose $\beta^{k+1} \leq \beta^{k}$.

(12) Set $k:=k+1$ and go to (1).

Observe that in this modification the iterations where the $\beta$-neighborhoods of the central path are reached are counted separately from the other iterations.

In order to obtain a polynomial complexity bound for the Modified LSSN algorithm we need to derive an upper bound for the number of iterations required by the algorithm to satisfy the central-path proximity condition given in step (6). The idea for bounding the number of iterations required by the algorithm to approach the central path is to study the line-search problem (26) and to find an upper bound for its objective function value. The next lemmas address these issues. 
Lemma 5.4 Let $\gamma \in(0,1), w=(x, y, z) \in \mathcal{F}_{\gamma}$, and $\mu \leq x^{T} z / n$.

Also let $\Delta w=(\Delta x, \Delta y, \Delta z)$ denote the solution of the system

$$
F^{\prime}(x, y, z)\left(\begin{array}{c}
\Delta x \\
\Delta y \\
\Delta z
\end{array}\right)=-F(x, y, z)+\mu\left(\begin{array}{l}
0 \\
0 \\
e
\end{array}\right)
$$

Consider $\phi=\frac{1}{\mu}\|\Delta X \Delta Z e\|_{2}$ where $\Delta X=\operatorname{diag}(\Delta x), \Delta Z=\operatorname{diag}(\Delta z)$.

Then, $w+\theta \Delta w \in \mathcal{F}_{\gamma}$ for $0 \leq \theta \leq \theta^{*}=\min (1,(1-\gamma) / \phi)$.

\section{Proof}

Let $w(\theta)=(x(\theta), y(\theta), z(\theta))=w+\theta \Delta w$. Then, we have that

$$
X(\theta) z(\theta)=(1-\theta) X z+\theta \mu e+\theta^{2} \Delta X \Delta z
$$

Since $w$ is strictly feasible $\Delta x^{T} \Delta z=0$ and from (27) we obtain

$$
x(\theta)^{T} z(\theta) / n=(1-\theta)\left(x^{T} z / n\right)+\theta \mu .
$$

For any $0 \leq \theta \leq 1$ we deduce that

$$
X(\theta) z(\theta) \geq \gamma(1-\theta)\left(x^{T} z / n\right) e+\theta \mu e-\theta^{2} \phi \mu e
$$

which implies

$$
X(\theta) z(\theta) \geq \gamma\left(x(\theta)^{T} z(\theta) / n\right) e+\theta[(1-\gamma)-\theta \phi] \mu e .
$$

It follows from (29) that if $0 \leq \theta \leq \theta^{*}=\min (1,(1-\gamma) / \phi)$ then $\frac{\min (X(\theta) Z(\theta) e)}{x(\theta)^{T} z(\theta) / n} \geq \gamma$. Now, let us show that

$$
x(\theta)>0, z(\theta)>0, \text { for all } 0 \leq \theta \leq \theta^{*}=\min (1,(1-\gamma) / \phi)
$$

For $\theta=0$ we have $w(0)=w$ and $(30)$ is satisfied because of the hypothesis that $w \in \mathcal{F}_{\gamma}$.

Let us suppose by contradiction that (30) is not satisfied for all $\theta \in\left[0, \theta^{*}\right]$. Then there exists $\hat{\theta} \in\left[0, \theta^{*}\right]$ and $\hat{i} \in\{1,2, \ldots, n\}$ such that either $x_{\hat{i}}(\hat{\theta})=0$ or $z_{\hat{i}}(\hat{\theta})=0$. But then from (29) we get

$$
0 \geq \gamma\left(x(\hat{\theta})^{T} z(\hat{\theta}) / n\right)+\hat{\theta}[(1-\gamma)-\hat{\theta} \phi] \mu
$$


From (28) we deduce that $x(\hat{\theta})^{T} z(\hat{\theta}) / n \geq \hat{\theta} \mu$ so that (31) gives the contradiction $0 \geq \gamma \hat{\theta} \mu$.

Lemma 5.4 states that the minimization problem (26) gives a lower value of the objective function than does the problem

$$
\min _{0 \leq \theta \leq \theta^{*}} f(\theta)
$$

The next lemma uses the result obtained from Lemma 5.4 and the optimization problem (32) in order to obtain an upper bound for the optimal value of (26). This upper bound will be used later for bounding the number of iterations needed to satisfy (25), i.e. the number of iterations needed for entering the $\beta$-neighborhood of the central path.

Lemma 5.5 Let $\gamma \in(0,2-\sqrt{2}], w=(x, y, z) \in \mathcal{F}_{\gamma}, \mu \leq x^{T} z / n$ and $\rho=\|(X z-\mu e) / \mu\|_{2}$. The optimal value of problem (26) is bounded above by

$$
\rho^{*}=\rho \max \left\{1-\frac{\gamma}{2 \sqrt{2}(1-\gamma)}, 1-\frac{\sqrt{2} \gamma(1-\gamma)}{\rho^{2}}\right\} .
$$

\section{Proof}

From (27) we deduce that

$$
\begin{gathered}
f(\theta)=\left\|(1-\theta) \frac{X z}{\mu}+\theta e+\theta^{2} \frac{\Delta X \Delta z}{\mu}-\epsilon\right\|_{2} \\
=\left\|(1-\theta)\left(\frac{X z}{\mu}-e\right)+\theta^{2} \frac{\Delta X \Delta z}{\mu}\right\|_{2} .
\end{gathered}
$$

As in the previous lemma, let us denote $\phi=\frac{1}{\mu}\|\Delta X \Delta Z e\|_{2}$. Then for any $0 \leq \theta \leq 1$ we obtain the inequality

$$
f(\theta) \leq(1-\theta)\left\|\frac{X z}{\mu}-e\right\|_{2}+\theta^{2} \phi .
$$

Let $\rho=\|(X z-\mu e) / \mu\|_{2}$ and $\rho(\theta)=(1-\theta) \rho+\theta^{2} \phi$. From (33) we have

$$
f(\theta) \leq \rho(\theta)
$$

Let

$$
\rho^{+}=\min _{0 \leq \theta \leq \theta^{*}} \rho(\theta) .
$$

It is clear from (34) and Lemma 5.4 that the the optimal value of problem (26) is bounded above by $\rho^{+}$. The remainder of the proof consists in finding an upper bound to $\rho^{+}$. 
Note that $\rho(0)=f(0)=\rho$. The unconstrained minimum of $\rho(\theta)=(1-\theta) \rho+\theta^{2} \phi$ is obtained for $\hat{\theta}=\rho /(2 \phi)$. We consider four cases.

(i) If $\rho \leq 2(1-\gamma)$ and $(1-\gamma) / \phi \leq 1$ then $\rho /(2 \phi)=\hat{\theta} \leq \theta^{*}=(1-\gamma) / \phi$ and consequently

$$
\rho^{+}=\rho(\hat{\theta})=\left(1-\frac{\rho}{4 \phi}\right) \rho .
$$

We want to find an upper bound for $\rho^{+}$in terms of $\rho$ and $\gamma$. To this effect we use the inequality

$$
\|\Delta X \Delta z\|_{2} \leq \frac{1}{2 \sqrt{2}}\left\|(X Z)^{-1 / 2}(X z-\mu e)\right\|^{2},
$$

whose proof can be found in [16]. According to the hypothesis of our lemma $w=(x, y, z) \in$ $\mathcal{F}_{\gamma}$ and $\mu \leq x^{T} z / n$ which leads us to the inequality

$$
\phi \leq \frac{\rho^{2}}{2 \sqrt{2} \gamma} .
$$

Substituting into (36) and using the fact that $\rho \leq 2(1-\gamma)$ we obtain the following bound for case (i):

$$
\rho^{+} \leq\left(1-\frac{\gamma}{2 \sqrt{2}(1-\gamma)}\right) \rho .
$$

(ii) On the other hand if $\rho \leq 2(1-\gamma)$ and $(1-\gamma) / \phi>1$ then $\theta^{*}=1$ and we may have either $\hat{\theta} \leq \theta^{*}=1$ in which case (36) and (38) hold, or $\hat{\theta}>\theta^{*}=1$ and then

$$
\rho^{+}=\rho(1)=\phi<\rho / 2 \text {. }
$$

Note that since $\gamma \leq 2-\sqrt{2}$ then $\rho / 2$ is less than the quantity on the right-hand side of (38), so that (38) holds whenever $\rho \leq 2(1-\gamma)$.

(iii) Now if we suppose that $\rho>2(1-\gamma)$ and $(1-\gamma) / \phi \leq 1$, then $\rho /(2 \phi)=\hat{\theta}>\theta^{*}=$ $(1-\gamma) / \phi$, which implies

$$
\rho^{+}=\rho\left(\theta^{*}\right)=\left(1-\frac{1-\gamma}{\phi}+\frac{(1-\gamma)^{2}}{\phi \rho}\right) \rho<\left(\frac{1-\gamma}{2 \phi}\right) \rho .
$$

Using (37) we obtain the upper bound

$$
\rho^{+} \leq\left(1-\frac{\sqrt{2} \gamma(1-\gamma)}{\rho^{2}}\right) \rho
$$


(iv) Finally, if we assume that $\rho>2(1-\gamma)$ and $(1-\gamma) / \phi>1$, then $\rho /(2 \phi)=\hat{\theta}>\theta^{*}=1$, and therefore (39) holds in this case as well. But then so does (38) and (41).

In conclusion, we have shown that (38) holds whenever $\rho \leq 2(1-\gamma)$, while (41) holds whenever $\rho>2(1-\gamma)$. We end the proof of our lemma by noting that the right-hand side of (41) is greater than the right-hand side of (38) if and only if $\rho>2(1-\gamma)$.

Lemma 5.6 Let $\left\{\left(x^{k, l}, y^{k, l}, z^{k, l}\right)\right\}$ and $\left\{\mu^{k}\right\}$ be generated by the Modified LSSN algorithm with the parameter choice $\left\{\beta^{k}\right\}$.

For each $k \geq 0$ let $l^{k}$ denote the iterate such that

$$
\left\|X^{k, l^{k}} z^{k, l^{k}} / \mu^{k}-e\right\|_{2} \leq \beta^{k}
$$

Then $l^{k}$ is well defined with

$$
l^{0}=O\left(n^{2} / \gamma\right) \text { and } l^{k}=O(n / \gamma)+O\left(\log \beta^{k}\right) \text { for } k \geq 1 .
$$

\section{Proof}

Let $\rho^{k, l}=\left\|X^{k, l} z^{k, l} / \mu^{k}-e\right\|_{2}$. From Lemma 5.5 we have that

$$
\rho^{k, l+1} \leq\left(1-\frac{\gamma}{2 \sqrt{2}(1-\gamma)}\right) \rho^{k, l} \text { if } \rho^{k, l} \leq 2(1-\gamma)
$$

and

$$
\rho^{k, l+1} \leq\left(1-\frac{\sqrt{2} \gamma(1-\gamma)}{\left(\rho^{k, l}\right)^{2}}\right) \rho^{k, l} \text { if } \rho^{k, l}>2(1-\gamma):
$$

Using (43) and the fact that $\rho^{k, j+1} \leq \rho^{k, j}$ we obtain the relation

$$
\rho^{k, l+1} \leq\left(1-\frac{\sqrt{2} \gamma(1-\gamma)}{\rho^{k, l} \rho^{k, 0}}\right) \rho^{k, l}=\left(\rho^{k, l}-\frac{\sqrt{2} \gamma(1-\gamma)}{\rho^{k, 0}}\right) .
$$

It follows that

$$
\rho^{k, l} \leq \rho^{k, 0}-l \frac{\sqrt{2} \gamma(1-\gamma)}{\rho^{k, 0}} .
$$

Therefore, $\rho^{k, l} \leq 2(1-\gamma)$ in at most

$$
\bar{l}^{k}=\frac{\left(\rho^{k, 0}-2(1-\gamma)\right) \rho^{k, 0}}{\sqrt{2} \gamma(1-\gamma)}
$$


steps.

From (42)

$$
\rho^{k, l^{k}+l} \leq\left(1-\frac{\gamma}{2 \sqrt{2}(1-\gamma)}\right)^{l} \rho^{k, l^{k}} \leq\left(1-\alpha^{k}\right)^{l} 2(1-\gamma)
$$

where $\alpha=\gamma /(2 \sqrt{2}(1-\gamma))$.

It follows that $\rho^{k, l^{k}+l} \leq \beta^{k}$ in at most

$$
\hat{l}^{k}=\frac{\log \left(\frac{\beta^{k}}{2(1-\gamma)}\right)}{\log (1-\alpha)}
$$

steps.

The following observations are in order. If $\rho^{k, 0}<2(1-\gamma) \leq \beta^{k}$ then $l^{k}=\bar{l}^{k}=\hat{l}^{k}=0$. If $2(1-\gamma)>\beta^{k}$ then $\vec{l}^{k}=0$ and $\hat{l}^{k}$ is given by (46).

On the other hand, if $\rho^{k, 0}>2(1-\gamma)$ and $2(1-\gamma) \leq \beta^{k}$ we have that $l^{k}=\bar{l}^{k}$ as defined by (45) and $\hat{l}^{k}=0$.

Hence, $l^{k}$ is well defined and from (45) and (46) we obtain that $l^{k} \leq \bar{l}^{k}+\hat{l}^{k}$.

The rest of the proof is devoted to finding bounds for $\rho^{k, 0}$.

For any $0 \leq \mu \leq\left(x^{0}\right)^{T} z^{0} / n$, it is clear that

$$
\left\|X^{0} z^{0}-\mu \epsilon\right\|_{2}^{2} \leq\left\|X^{0} z^{0}\right\|_{2}^{2} \leq\left(\left(x^{0}\right)^{T} z^{0}\right)^{2}
$$

Let $\mu^{0}=\sigma^{0}\left(x^{0}\right)^{T} z^{0} / n$. It follows from (47) that $\rho^{0,0} \leq n / \sigma^{0}$.

According to (45), this implies that

$$
l^{0}=O\left(n^{2} / \gamma\right)
$$

If $\beta^{0}$ is independent of $n$, say $\beta^{0}=\frac{1}{4}$, then

$$
\hat{l}^{0}=O(1)
$$

so that

$$
l^{0}=O\left(n^{2} / \gamma\right)
$$

Now,

$$
\left(\rho^{k, 0}\right)^{2}=\left\|X^{k} z^{k} / \mu^{k}-e\right\|_{2}^{2}=\frac{1}{\left(\mu^{k}\right)^{2}}\left\|X^{k} z^{k}-\mu^{k} e\right\|_{2}^{2}
$$




$$
=\frac{1}{\left(\mu^{k}\right)^{2}}\left(\left\|X^{k} z^{k}-\left(x^{k^{T}} z^{k} / n\right) e\right\|_{2}^{2}+\left(\mu^{k}-x^{k^{T}} z^{k} / n\right)^{2}\|e\|_{2}^{2}\right) .
$$

By definition of the Modified LSSN algorithm,

$$
\left\|X^{k} z^{k}-\mu^{k-1} e\right\|_{2} \leq \beta^{k-1} \mu^{k-1}, \text { for each } k \geq 1,
$$

and because $\left(x^{k^{T}} z^{k} / n\right) e$ is the orthogonal projection of $X^{k} z^{k}$ over the subspace generated by $e$ we have,

$$
\left\|X^{k} z^{k}-\left(x^{k^{T}} z^{k} / n\right) e\right\|_{2} \leq\left\|X^{k} z^{k}-\mu^{k-1} e\right\|_{2} .
$$

Since $\mu^{k}=\sigma^{0} x^{k^{T}} z^{k} / n$ we obtain

$$
\begin{gathered}
\left(\rho^{k, 0}\right)^{2} \leq \frac{1}{\left(\mu^{k}\right)^{2}}\left(\left(\beta^{k-1}\right)^{2}\left(x^{k^{T}} z^{k} / n\right)^{2}+n\left(\mu^{k}-x^{k^{T}} z^{k} / n\right)^{2}\right) \\
=\frac{1}{\left(\mu^{k}\right)^{2}}\left(\left(\beta^{k-1}\right)^{2}\left(x^{k^{T}} z^{k} / n\right)^{2}+n\left(1-\sigma^{0}\right)^{2}\left(x^{k^{T}} z^{k} / n\right)^{2}\right) .
\end{gathered}
$$

It follows that

$$
\left(\rho^{k, 0}\right)^{2} \leq \frac{\left(\beta^{*}\right)^{2}+\left(1-\sigma^{0}\right)^{2} n}{\left(\sigma^{0}\right)^{2}}
$$

where $\beta^{*}=\max _{k \geq 0} \beta^{k}$.

Finally, from (45), (46), (53), and $l^{k} \leq \bar{l}^{k}+\hat{l}^{k}$ we deduce that $l^{k}=O(n / \gamma)+O\left(\log \beta^{k}\right)$, for $k \geq 1$.

The previous lemma gives an upper bound for the number of iterations required by the Modified LSSN algorithm to enter the $\beta$-neighborhood of the central path. The polynomial complexity bound for the algorithm is obtained by assuming a certain rate of decrease for $\beta^{k}$ and by finding a bound on the number of iterations required by the algorithm to reduce the gap to a given tolerance. This is done in the next theorem.

Theorem 5.2 Let $\left\{\left(x^{k}, y^{k}, z^{k}\right)\right\}$ and $\left\{\mu^{k}\right\}$ be generated by the Modified LSSN algorithm with $\left\{\left(x^{0}, y^{0}, z^{0}\right)\right\}$ a strictly feasible point and the parameter choices $\left\{\beta^{k}\right\}$.

Choose $\epsilon>0$ and $\sigma^{0} \in(0,1)$ such that $\sigma^{0}\left(\beta^{*}+1\right)<1$ where $\beta^{*}=\max _{k \geq 0} \beta^{k}$. Then,

(i) $x^{k^{T}} z^{k} \leq \epsilon$ in at most $k_{\epsilon}$ steps, with $k_{\epsilon}=O\left(\log \left(x^{0}\right)^{T} z^{0} / \epsilon\right)$. 
(ii) Let $\beta^{k}$ be chosen as $\beta^{k}=\left(\beta^{0}\right)^{k}$. Then, the Modified LSSN algorithm iteration complexity is

$$
O\left(n^{2} / \gamma\right)+O\left(\left[n / \gamma+\log \frac{\left(x^{0}\right)^{T} z^{0}}{\epsilon}\right] \log \frac{\left(x^{0}\right)^{T} z^{0}}{\epsilon}\right) .
$$

(iii) Let $\beta^{k}$ be chosen as $\beta^{k}=\frac{1}{2(k+1)}$. Then, the Modified LSSN algorithm iteration complexity is

$$
O\left(n^{2} / \gamma\right)+O\left(\left[n / \gamma+\log \left(\log \frac{\left(x^{0}\right)^{T} z^{0}}{\epsilon}\right)\right] \log \left(\frac{\left(x^{0}\right)^{T} z^{0}}{\epsilon}\right) .\right.
$$

\section{Proof}

From (51) we have that $x_{i}^{k+1} z_{i}^{k+1} \leq\left(1+\beta^{k}\right) \mu^{k}=\left(1+\beta^{k}\right) \sigma^{0} x^{k^{T}} z^{k} / n$. for all $k \geq 0$. Therefore, $x^{k^{T}} z^{k} \leq \eta^{k}\left(x^{0}\right)^{T} z^{0}$ where $\eta=\left(1+\beta^{*}\right) \sigma^{0}$ with $\beta^{*}=\max _{k \geq 0} \beta^{k}$ and $\eta<1$ by hypothesis. Hence, $x^{k^{T}} z^{k} \leq \epsilon$ in at most $k_{\epsilon}=O\left(\log \left(x^{0}\right)^{T} z^{0} / \epsilon\right)$.

From Lemma 5.6

$$
\sum_{k=1}^{k_{\epsilon}} l^{k}=O\left(\frac{n}{\gamma} \log \left(\frac{\left(x^{0}\right)^{T} z^{0}}{\epsilon}\right)\right)+O\left(\log \left(\frac{\left(x^{0}\right)^{T} z^{0}}{\epsilon}\right)^{2}\right) \text { if } \beta^{k}=\left(\beta^{0}\right)^{k}
$$

and

$$
\sum_{k=1}^{k_{\epsilon}} l^{k}=O\left(\frac{n}{\gamma} \log \left(\frac{\left(x^{0}\right)^{T} z^{0}}{\epsilon}\right)\right)+O\left(\log \left(\frac{\left(x^{0}\right)^{T} z^{0}}{\epsilon}\right) \log \left(\log \left(\frac{\left(x^{0}\right)^{T} z^{0}}{\epsilon}\right)\right) \text { if } \beta^{k}=\frac{1}{2(k+1)} .\right.
$$

Items (ii) and (iii) follow from (54), (55) and the fact that $l^{0}=O\left(n^{2} / \gamma\right)$ (see Lemma 5.5).

Corollary 5.1 Consider problem (1) and assume the entries of the data $A, b, c$ are integers with $L$ being the bit length of that input data.

Let $\left\{\left(x^{0}, y^{0}, z^{0}\right)\right\}$ be a strictly feasible point such that $\left(x^{0}\right)^{T} z^{0} \leq 2^{2 L}$.

Then, iteration complexity of the Modified LSSN algorithm is

$$
O\left(n^{2} / \gamma\right)+O([n / \gamma+L] L), \quad \text { if } \beta^{k}=C \beta^{k-1} \text { for } k \geq 1 \text { and for some } C \in(0,1)
$$

and

$$
O\left(n^{2} / \gamma\right)+O([n / \gamma+\log (L)] L), \quad \text { if } \beta^{k}=O\left(\frac{1}{k}\right) \text { for } k \geq 1 .
$$

Proof Let $\epsilon=2^{-2 L}$. The proof follows directly from Theorem 5.2 since $\log \frac{\left(x^{0}\right)^{T} z^{0}}{\epsilon} \leq 4 L$. 


\section{Numerical Experience}

In this section we discuss the numerical results obtained from applying the LSSN algorithm to a subset of the NETLIB test problems. We compare the performance of the LSSN algorithm with the performance of the Kojima-Mizuno-Yoshise algorithm and the Mizuno-Todd-Ye algorithm.

Our preliminary numerical experimentation showed that the LSSN algorithm as presented in Section 3 (Algorithm 3.1) seems to perform better than the Modified LSSN algorithm in terms of number of iterations. Therefore, the results in the present section use the LSSN algorithm without modification (Algorithm 3.1). At this juncture we believe that the linesearch modification introduced in Section 5 has more of a theoretical yalue than a practical value. However, more research will be done on this issue.

\subsection{Implementation Issues}

The experiments were performed in 64 bit arithmetic on a Sun 4/model 670-120 using codes implemented in MATLAB.

\subsubsection{Stopping Criteria}

The algorithm is terminated when

$$
\max \left(\frac{\left|c^{T} x^{k}-b^{T} y^{k}\right|}{1+\left|b^{T} y^{k}\right|}, \frac{\left\|A x^{k}-b\right\|_{1}}{1+\|x\|_{1}}, \frac{\left\|A^{T} y^{k}+z^{k}-c\right\|_{1}}{1+\left\|y^{k}\right\|_{1}+\left\|z^{k}\right\|_{1}}, \frac{\left\|X^{k} z^{k}-e\right\|_{2}}{\left(x^{k}\right)^{T} z^{k} / n}\right) \leq 10^{-8},
$$

or when the number of iterations reaches 200 .

\subsubsection{Parameter Choices.}

The line-search strategy (backtracking) defined in step (4) of the LSSN algorithm was implemented using the value $\eta=10^{-4}$ and a fixed value $\rho=1 / 2$.

In all problems, the parameters $\tau^{k}$ were chosen as $\tau^{k}=1-\min \left\{0.05,0.05\left(x^{k}\right)^{T} z^{k}\right\}$. Therefore $\tau^{k}=0.95$ far away from the solution set and is closer to 1 when the duality gap is small. 
The step-lengths for the Mizuno-Todd-Ye algorithm were obtained following Ye, Güler, Tapia, and Zhang [20].

The parameters $\beta^{j}$ were chosen, in general, as $\beta^{k+1}=\left(\beta^{k}\right)^{2}$. Some minor variations, based on heuristics, were considered in choosing $\beta^{j}$ when the iterates were close to the solution set. In the numerical results presented in this section $\beta=\beta^{0}=0.25$ for the Mizuno-ToddYe and LSSN algorithms. The choice of $\beta^{0}=0.25$ was made so that we could compare the Mizuno-Todd-Ye algorithm with the LSSN algorithm. Our experience has shown that numerical efficiency for the LSSN algorithm can be substantially improved by choosing $\beta$ significantly larger than 0.25 .

\subsubsection{Starting iterates}

The starting points for the LSSN and Kojima-Mizuno-Yoshise algorithms are obtained following Lustig, Marsden, and Shanno [12] and are not necessarily feasible.

The algorithms generate a sequence of iterates that approach feasibility and have the property that the duality gap goes to zero. For the LSSN algorithm, the infeasibility of the starting iterates should be considered a computational convenience since in all the problems tested when the initial $\beta$-neighborhood of the central path is entered for the very first time the iterates are already feasible. Thus, equivalent experimentation could have been done starting the algorithm from the strictly feasible iterates obtained in the process.

A very interesting feature of the LSSN algorithm is that it provides a way of computing a point in a $\beta$-neighborhood of the central path, for any $\beta \in(0,1)$. In this work we used this feature of the LSSN algorithm for computing the starting point for the Mizuno-Todd-Ye algorithm.

\subsection{Numerical Results}

Our numerical results are summarized in the following three tables. Table 1 studies the effect of the choice of the parameter $\sigma^{0}$ in the performance of the LSSN algorithm. Table 2 compares the performance of the LSSN and the Mizuno-Todd-Ye algorithms. The starting point is the same for both algorithms and the iterations are counted from that point. Table 3 shows the performance of the Kojima-Mizuno-Yoshise algorithm for fixed values of the 
centering parameters $\sigma^{k}$.

Observe that the worst performance of the LSSN algorithm is obtained for very small or very large values of $\sigma^{0}$. On one hand, if the parameter $\sigma^{0}$ is small (e.g. $\sigma^{0} \leq 0.0001$ for most of the problems we considered) the number of iterations exceeds the allowable maximum number of 200 iterations. This large number of iterations is due to the fact that the iterates approach the solution set very fast but are still far away from the central path. Because of the proximity of the iterates to the solution set the Jacobian matrix is very ill-conditioned and the step-lengths become very small. On the other hand, comparing the values of $\sigma^{0}$ for which the algorithm converges, it can be observed that the closer the value of the parameter $\sigma^{0}$ is to 1 , the stronger is the adherence of the iterates to the central path and the slower is the performance of the algorithm (as should be expected from the theory). Observe from Table 1 that $\sigma^{0}$ equal to 0.01 seems to be a good choice to use in implementations.

Notice that the number of iterations required to enter the initial $\beta$-neighborhood does not seem to follow, in general, a constant pattern with respect to the values of $\sigma^{0}$. However, when the line-search strategy is activated, the larger the value of $\sigma^{0}$ the larger is the number of times the step must be decreased (by backtracking). We believe this behavior reflects the fact that the larger the value of $\sigma^{0}$, the more difficult it is for the LSSN algorithm to obtain appropiate decrease in the merit function, since a higher priority is placed on centrality.

For most of the problems tested, the Mizuno-Todd-Ye algorithm requires a larger number of linear systems to be solved to converge than does the LSSN algorithm, as can be observed from Table 2. Recall the cost per iteration of the Mizuno-Todd-Ye algorithm is twice that of the LSSN algorithm. Actually, for many of the problems the Mizuno-Todd-Ye algorithm also requires a larger number of iterations to converge than does the LSSN algorithm. This result seems to be a consequence of the long steps performed by the LSSN algorithm. An illustration of the behavior of the Mizuno-Todd-Ye and LSSN algorithms with respect to closeness to the central path and the duality gap is given by Figures 1 and 2 for problem SHARE2B.

Finally, Table 3 shows that the Kojima-Mizuno-Yoshise algorithm seems not to be converging for several of the problems considered. This is a significant disadvantage when compared with the LSSN and the Mizuno-Todd-Ye algorithns. The lack of convergence of the Kojima-Mizuno-Yoshise algorithm is due to the fact that the iterates do not stay in 
Table 1: Study of the effect of different choices of the parameter $\sigma^{0}$ for the LSSN algorithm.

\begin{tabular}{|c|c|c|c|c|c|c|}
\hline PROBLEM & $a^{0}$ & $\mathbf{k}^{1}$ & $\mathbf{k e p}^{2}$ & $18^{3}$ & $f c p^{4}$ & \\
\hline \multirow[t]{6}{*}{ AFIRO } & 0.00001 & 20 & 15 & 0 & 2 & \\
\hline & 0.0001 & 21 & 13 & 0 & 3 & \\
\hline & 0.001 & 22 & 11 & 0 & 3 & \\
\hline & 0.01 & 20 & 7 & 0 & 3 & \\
\hline & 0.1 & 25 & 8 & 0 & 5 & \\
\hline & 0.5 & 65 & 8 & 0 & 18 & \\
\hline \multirow[t]{7}{*}{ BLEND } & 0.00001 & $\mathrm{NG}$ & - & - & - & \\
\hline & 0.0001 & NC & - & - & - & \\
\hline & 0.001 & $\mathrm{NC}$ & - & - &.- & \\
\hline & 0.01 & 30 & 12 & 0 & 3 & \\
\hline & 0.1 & 40 & 12 & 2 & 6 & \\
\hline & 0.3 & 71 & 25 & 34 & 11 & \\
\hline & 0.5 & 119 & 50 & 132 & 19 & \\
\hline \multirow[t]{6}{*}{ SCSD1 } & 0.00001 & $\mathrm{NC}$ & - & - & - & \\
\hline & 0.0001 & $\mathrm{NC}$ & - & - & - & \\
\hline & 0.001 & $\mathrm{NC}$ & - & - & - & \\
\hline & 0.01 & 25 & 4 & 0 & 4 & \\
\hline & 0.1 & 34 & 3 & 0 & 7 & \\
\hline & 0.5 & 76 & 3 & 0 & 21 & \\
\hline \multirow[t]{6}{*}{ SHARE2B } & 0.00001 & 28 & 21 & 0 & 2 & \\
\hline & 0.0001 & $\mathrm{NC}$ & - & - & - & \\
\hline & 0.001 & 28 & 13 & 0 & 3 & \\
\hline & 0.01 & 3.3 & 12 & 0 & 3 & \\
\hline & 0.1 & 41 & 13 & 0 & 6 & \\
\hline & 0.5 & 121 & 57 & 145 & 17 & \\
\hline \multirow[t]{6}{*}{ SCTAP1 } & 0.00001 & NO & - & - & - & \\
\hline & 0.0001 & NC: & - & - & - & \\
\hline & 0.001 & NC: & - & - & - & \\
\hline & 0.01 & 44 & 10 & 0 & 4 & \\
\hline & 0.1 & 61 & 18 & 17 & 7 & \\
\hline & 0.5 & 123 & 39 & 81 & 20 & \\
\hline \multirow[t]{6}{*}{ LOTFI } & 0.00001 & $\mathrm{NC}$ & - & - & - & \\
\hline & 0.0001 & NC & - & - & - & \\
\hline & 0.001 & NC: & - & - & - & \\
\hline & 0.01 & 96 & 61 & 86 & 4 & \\
\hline & 0.1 & 109 & 63 & 87 & 8 & \\
\hline & 0.5 & 180 & 70 & 155 & 21 & \\
\hline \multirow[t]{6}{*}{ SCAGR7 } & 0.00001 & 33 & 21 & 0 & 2 & \\
\hline & 0.0001 & 35 & 18 & 0 & 3 & \\
\hline & 0.001 & 32 & 13 & 0 & 3 & \\
\hline & 0.01 & 36 & 11 & 0 & 4 & \\
\hline & 0.1 & 46 & 10 & 0 & 7 & \\
\hline & 0.5 & 77 & 10 & 0 & 16 & - \\
\hline \multirow[t]{6}{*}{ SCAGF25 } & 0.00001 & 43 & 32 & 0 & 2 & \\
\hline & 0.0001 & 40 & 20 & 0 & 3 & \\
\hline & 0.001 & 40 & 20 & 0 & 3 & \\
\hline & 0.01 & 37 & 14 & 0 & 3 & \\
\hline & 0.1 & 45 & 11 & 0 & 6 & \\
\hline & 0.5 & 77 & 11 & 0 & 16 & \\
\hline \multicolumn{7}{|c|}{${ }^{1}$ Number of Iterations. } \\
\hline \multicolumn{7}{|c|}{$\begin{array}{l}2 \text { Number of iterations taken to enter the initial } \beta \text { neighborhood of the central path. } \\
{ }^{3} \text { Number of times the linesearch strategy decreases the step length. }\end{array}$} \\
\hline
\end{tabular}


Table 2: Comparison between the Mizuno-Todd-Ye and LSSN algorithms in terms of number of linear systems solved.

\begin{tabular}{|l|c|c|c|}
\hline PROBLEM & M-T-Y & LSSN & $\sigma^{0}$ \\
\hline \hline AFIRO & 16 & 11 & 0.001 \\
& 28 & 13 & 0.01 \\
& 32 & 17 & 0.1 \\
\hline BLEND & 52 & 18 & 0.01 \\
& 54 & 28 & 0.1 \\
& 54 & 69 & 0.5 \\
\hline SCSD1 & 62 & 21 & 0.01 \\
& 70 & 73 & 0.5 \\
\hline SHAFE2B & 18 & 15 & 0.00001 \\
& 42 & 15 & 0.001 \\
& 58 & 21 & 0.01 \\
& 62 & 28 & 0.1 \\
& 64 & 64 & 0.5 \\
\hline SCTAP1 & 122 & 43 & 0.1 \\
& 90 & 34 & 0.01 \\
\hline LOTFI & 106 & 46 & 0.1 \\
\hline SCAGR7 & 52 & 19 & 0.001 \\
& 84 & 67 & 0.5 \\
\hline SCAGR25 & 80 & 34 & 0.1 \\
\hline SDSD6 & NC & 47 & 0.1 \\
\hline
\end{tabular}

1 The allowable maximum number of 200 iterations is reached

Table 3: Performance of the Kojima-Mizuno-Yoshise algorithm.

\begin{tabular}{|l|c|c|}
\hline PROBLEM & Number of Iterations & $\sigma^{k}$ \\
\hline \hline AFIRO & $\mathrm{NC}^{1}$ & 0.3 \\
& 39 & 0.5 \\
& 105 & 0.8 \\
\hline BLEND & $\mathrm{NC}$ & 0.3 \\
& $\mathrm{NC}$ & 0.5 \\
& $\mathrm{NC}$ & 0.8 \\
\hline SHARE2B & $\mathrm{NC}$ & 0.3 \\
& $\mathrm{NC}$ & 0.5 \\
& $\mathrm{NC}$ & 0.8 \\
\hline LOTFI & NC & 0.5 \\
& $\mathrm{NC}$ & 0.8 \\
\hline SCAGR7 & 39 & 0.3 \\
& 56 & 0.5 \\
& 146 & 0.8 \\
\hline SCAGR25 & 41 & 0.3 \\
& 53 & 0.5 \\
& 138 & 0.8 \\
\hline \multicolumn{2}{|c|}{ The allowable maximum number of 200 iterations is reached. }
\end{tabular}


or return to a $\beta$-neighborhood of the central path after such a neighborhood is reached. The poor performance of the Kojima-Mizuno-Yoshise algorithm is a consequence of the fact that our stopping criteria reflects convergence to the analytic center, and apparently this algorithm does not in general converge to the analytic center.

In the problems where the Kojima-Mizuno-Yoshise algorithm converges, the number of iterations increases as the value of the parameter $\sigma^{k}$ increases, as should be expected. The smallest number of iterations required by the Kojima-Mizuno-Yoshise algorithm to converge is always larger than the smallest number of iterations required by the LSSN algorithm to converge for the same problem. It is very interesting to note that for those problems where the Kojima-Mizuno-Yoshise algorithm converges, the line-search strategy is never activated when applying the LSSN algorithm, i.e. they seemed to be rather easy problems. In fact, we have found out that those problems share the feature of having a unique primal solution.

\section{Conclusions}

In this work a modification of the Kojima-Mizuno-Yoshise primal-dual algorithm is proposed. The goals of approaching the central path and the solution set are combined to design an algorithm which effectively computes the analytic-center solution. The iterates generated by the Long-Step Shrinking-Neighborhood (LSSN) algorithm proposed in this research approach the solution set while approaching the central path. The approach to the central path is done in such a way that we attempt to take full Newton steps (long steps) as much as possible. Two aspects of the algorithm facilitate this objective. Firstly, when the duality gap is large we choose $\beta$-neighborhoods that are large. Secondly, once we reach a $\beta$-neighborhood our subsequent step is not required to stay in a $\beta$-neighborhood, i.e. is not required to stay near the central path. However, as might be expected from the Gonzaga and Tapia [8] convergence theory for the Mizuno-Todd-Ye algorithm, near the solution when the duality gap is small full Newton steps actually stay close to the central path and often fall in our chosen $\beta$-neighborhoods; eventhough the $\beta$-neighborhoods are shrinking.

Let us now briefly make some comments concerning shrinking $\beta$-neighborhoods versus fixed $\beta$-neighborhoods as employed in the Mizuno-Todd-ye algorithm. It is clear that far 
from the solution when the duality gap is large it is not computationally effective to enforce excessive fidelity to the central path; hence we should allow for large $\beta$-neighborhoods in this situation. Our convergence theory given in Section 4 for the algorithm described in Section 3 is based on the Zhang-Tapia [24] sufficient condition for convergence to the analytic center. This theory requires the satisfaction of condition (a2) given in (6); which says that for the shrinking $\beta$-neighborhoods we must have the sequence of $\beta$ 's converging to zero. It is also worth noting that our polynomial bound complexity theory given in Section 5 requires the sequence of $\beta$ 's to converge to zero. Quite recently Bonnans and Gonzaga. [1] derived an elegant theory giving conditions that are sufficient for convergence to the analytic center. Their theory is less restrictive than the Zhang-Tapia [24] theory and does not require the sequence of $\beta$ 's to converge to zero. Their theory suggests that in our algorithm, near the solution when the duality gap is small $\beta$ can be held fixed. Very recently while this paper was in review we performed some preliminary numerical investigations with a version of our algorithm which left $\beta$ fixed near the solution. The results were quite satisfactory. Hence it seems as if the Bonnans-Gonzaga theory could be used to establish convergence to the analytic center for a version of our algorithm that allows fixed $\beta$ when the duality gap is small. Moreover, the Bonnans-Potra [2] convergence theory, which extends the BonnansGonzaga theory to infeasible iterates, could be used to analyze our algorithm in the case of infeasible iterates. Recall, as we previously mentioned, that in all our numerical experiments feasibility was established in the first $\beta$-neighborhood pass and the iterates remained feasible in subsequent $\beta$-neighborhood passes. Hence, the theory presented in Section 4 is applicable.

A very interesting feature of the LSSN algorithm is that it gives a practical way of finding a point in a neighborhood of the central path. Hence, the LSSN algorithm can be used for finding a starting point for the Mizuno-Todd-Ye algorithm and comparisons of the performances of the Generic Kojima-Mizuno-Yoshise, Mizuno-Todd-Ye, and LSSN algorithms have been established.

Numerical results showed that the Generic Kojima-Mizuno-Yoshise algorithm is not adequate for computing the analytic-center solution even if the centering parameters $\sigma^{k}$ are chosen close to one and the iterates reach the central path at some iteration. The MizunoTodd-Ye algorithm and the LSSN algorithm are, in general, both capable of computing the analytic-center. The LSSN algorithm compares favorably to the Mizuno-Todd-Ye algorithm 
regarding total cost. In this work we proposed the use of the LSSN algorithm for finding the starting point for the Mizuno-Todd-Ye algorithm, but different approaches of obtaining this initial point may lead to better performance of the Mizuno-Todd-Ye algorithm. The performance of the LSSN algorithm strongly depends on the choice of the parameter $\sigma^{0}$. The performance of the Mizuno-Todd-Ye algorithm depends on the starting point and does not involve parametric choices, thus, in this sense, the Mizuno-Todd-Ye algorithm is less complicated than the LSSN algorithm. However, it was our experience that while in theory the Mizuno-Todd-Ye algorithm is guaranteed to converge to the analytic center, in practice this is not necessarily the case (obviously due to the ill-conditioning encountered near the solution set).

\section{Acknowledgment}

The authors would like to thank Rongqin Sheng for carefully reading the manuscript and suggesting several improvements. They also thank Amr El-Bakry for numerous discussions, and two anonymous referees for constructive comments.

\section{References}

[1] BONNANS,J. F., and GONZAGA, C. C. Convergence of Interior Point Algorithms for the Monotone linear Complementarity Problem. Unpublished manuscript, Oct. 1993.

[2] BONNANS,J. F., and POTRA, F. A. On the convergence of the iteration sequence of infeasible path following algorithms for linear complementarity problems. Unpublished manuscript, Oct. 1995.

[3] CHARNES, A., COOPER, W.W., and THRALL, R.M. A structure for Classifying and Characterizing Efficiency and Inefficiency in Data Envelopment Analysis. The Journal of Productivity Analysis, Vol. 2, pp.197-237, 1991.

[4] DENNIS, J. E., SCHNABEL, R. B. Numerical methods for Unconstrained Optimization and Nonlinear Equations. Prentice-Hall, Inc., Englewood Cliffs, NJ., 1983.

[5] EL-BAKRY, A., TAPIA, R. A., TSUCHIYA, T., ZHANG, Y. On the formulation of the Primal-Dual Newton Interior-Point Method for Nonlinear Programming. Technical 
Report TR92-40, Dept. Mathematical Sciences, Rice University, 1992. To appear in J. Optimization Theory and Applications.

[6] EL-BAKRY, A., TAPIA, R. A., and ZHANG, Y. On the use of indicators in identifying zero variables for interior point methods. SIAM Review, Vol. 36, Number 1, pp. 45-72, 1994.

[7] GONZAGA, C. C. Path-Following Methods for Linear Programming. SIAM Review, Vol. 34, pp.167-224, 1992.

[8] GONZAGA, C. C., and TAPIA, R. A. On the convergence of the Mizuno-Todd-Ye algorithm to the analytic center of the solution set. Technical Report TR92-41, Dept. Mathematical Sciences, Rice University, 1992, to appear in SIAM journal on Optimization.

[9] GONZALEZ-LiMA, M. D. Effective Computation of the Analytic Center of the Solution Set in Linear Programming Using Primal-Dual Interior-Point Methods. Rice University, Dept. of Computational and Applied Mathematics, TR94-48, 1994.

[10] GULER, O. and YE, Y. Convergence behavior of some interior-point algorithms. Mathematical Programming, 60:215-228, 1993.

[11] KOJIMA, M., MIZUNO, S., and YOSHISE, A. A primal-dual interior point method for linear programming. Progress in Mathematical programming, interior-point and related methods, Nimrod Megiddo editor, pp. 29-47. Springer-Verlag, New York, 1989.

[12] LUSTIG, I., MARSTEN, R., SHANNO, D. On implementing Mehrotra's PredictorCorrector Interior Point method for linear programming. SIAM Journal on Optimization, 2, pp. 435-449, 1992.

[13] McLindEN, L. An analogue of Moreau's approximation theorem, with application to the nonlinear complementarity problem. Pacific J. of Math., Vol. 88, pp.101-161, 1980.

[14] McSHANE, K. A superlinearly convergent $O(\sqrt{n} L)$-iteration interior point algorithms for linear programming and the monotone linear complementarity problem. SIAM Journal on Optimization, 4(2), pp. 247-261, 1994. 
[15] MEGIDDO, N. Pathways to the optimal set in linear programming. Edited by N. Megiddo, Progress in Mathematical programming, interior-point and related methods, pp.131-158. Springer-Verlag, New York, 1989.

[16] MIZUNO, S., TODD, M. J. and YE, Y. On adaptive-step primal-dual interior-point algorithms for linear programming. Mathematics of Operations Research, 18:964-981, 1993.

[17] SONNEVEND, G. An analytic center for polyhedrons and new classes of global algorithms for linear (smooth, convex) programming. Lecture Notes in Control and Inf. Sci., A. Prekopa editor, Vol. 84:866-876. Springer, Berlin, 1985.

[18] TAPIA, R. A., ZHANG, Y. and YE, Y. On the convergence of the iteration sequence in primal-dual interior-point methods. Mathematical Programming 68, 1995, pp. 141-154.

[19] THOMPSON, R., DHAMARPALA, P.S., THRALL, R.M. DEA Sensitivity Analysis of Efficiency Measures with an Application to Kansas Farming and Illinois Coal Mining. Data Envelopment Analysis: Theory, Methodology and Applications, A. Charnes, W. W. Cooper, A. Y. Lewin and L. M. Seiford, eds., Kluwer, Boston, 1994, Chapter 20, pp. 293-422.

[20] YE, Y., GULER, O., TAPIA, R. A. and ZHANG, Y. A quadratically convergent $O(\sqrt{n} L)$-iteration algorithm for linear programming. Mathematical Programming 59, 1993, pp. 151-162.

[21] YE, Y., TAPIA, R. A. and ZHANG, Y. A superlinearly convergent $O(\sqrt{n} L)$-iteration algorithm for linear programming. Technical Report TR91-22, Dept. Mathematical Sciences, Rice University, 1991.

[22] ZHANG, Y. and EL-BAKRY, A. A modified predictor-corrector algorithm for locating weighted centers in linear programming. J. Optimization Theory and Appliations, Vol. 80, No. 2, 319-331, Feb. 1994. 
[23] ZHANG, Y. and TAPIA, R. A. Superlinear and quadratic convergence of primal-dual interior point methods for linear programming revisited. Journal of Optimization Theory and Applications 73 (1992), pp. 229-242.

[24] ZHANG, Y. and TAPIA, R. A. On the convergence of interior-point methods to the center of solution set in linear programming. Technical Report TR91-30, Dept. Mathematical Sciences, Rice University, 1991.

[25] ZHANG, Y., TAPIA, R. A. and DENNIS, J. E. On the superlinear and quadratic convergence of primal-dual interior point linear programming algorithms. SIAM Journal on Optimization 2, 1992, pp. 303-324.

[26] ZHANG, Y., TAPIA, R. A. and POTRA, F. On the superlinear convergence of interior point algorithms for a general class of problems. SIAM Journal on Optimization 3, 1993, pp. 413-422. 